\title{
Projective parameterized linear codes
}

\author{
Manuel González Sarabia, Carlos Rentería Márquez and Eliseo \\ Sarmiento Rosales
}

\begin{abstract}
In this paper we estimate the main parameters of some evaluation codes which are known as projective parameterized codes. We find the length of these codes and we give a formula for the dimension in terms of the Hilbert function associated to two ideals, one of them being the vanishing ideal of the projective torus. Also we find an upper bound for the minimum distance and, in some cases, we give some lower bounds for the regularity index and the minimum distance. These lower bounds work in several cases, particularly for any projective parameterized code associated to the incidence matrix of uniform clutters and then they work in the case of graphs.
\end{abstract}

\section{Introduction}

Let $K=\mathbb{F}_{q}$ be a finite field with $q$ elements and $L=K\left[Z_{1}, \ldots, Z_{n}\right]$ be a polynomial ring over the field $K$. Let $Z^{a_{1}}, \ldots, Z^{a_{m}}$ be a finite set of monomials. As usual if $a_{i}=\left(a_{i 1}, \ldots, a_{i n}\right) \in \mathbb{N}^{n}$, where $\mathbb{N}$ stands for the nonnegative integers, then we set

$$
Z^{a_{i}}=Z_{1}^{a_{i 1}} \ldots Z_{n}^{a_{i n}} \text { for all } i=1, \ldots, m .
$$

Key Words: Parameterized code, projective space, minimum distance, regularity index. 2010 Mathematics Subject Classification: Primary 13P25, 14G50; Secondary 14G15, 11T71, 94B27, 94B05.

Received: February, 2013

Revised: October, 2013.

Accepted: October, 2013. 
Consider the following set parameterized by these monomials

$$
X=\left\{\left[\left(t_{1}^{a_{11}} \cdots t_{n}^{a_{1 n}}, \ldots, t_{1}^{a_{m 1}} \cdots t_{n}^{a_{m n}}\right)\right] \in \mathbb{P}^{m-1} \mid t_{i} \in K^{*}\right\},
$$

where $K^{*}=K \backslash\{0\}$ and $\mathbb{P}^{m-1}$ is a projective space over the field $K$. Following [17] we call $X$ an algebraic toric set parameterized by $Z^{a_{1}}, \ldots, Z^{a_{m}}$. The set $X$ is a multiplicative group under componentwise multiplication.

In the same way, let $A$ be the $n \times m$ matrix given by

$$
\left(\begin{array}{cccc}
a_{11} & a_{21} & \cdots & a_{m 1} \\
a_{12} & a_{22} & \cdots & a_{m 2} \\
\vdots & \vdots & \vdots & \vdots \\
a_{1 n} & a_{2 n} & \cdots & a_{m n}
\end{array}\right)
$$

We say that the set defined in (1) is an algebraic toric set associated to the matrix $A$. We note that

$$
\begin{gathered}
{\left[\left(t_{1}^{a_{11}} \cdots t_{n}^{a_{1 n}}, t_{1}^{a_{21}} \cdots t_{n}^{a_{2 n}}, \ldots, t_{1}^{a_{m 1}} \cdots t_{n}^{a_{m n}}\right)\right]=} \\
{\left[\left(1, t_{1}^{a_{21}-a_{11}} \cdots t_{n}^{a_{2 n}-a_{1 n}}, \ldots, t_{1}^{a_{m 1}-a_{11}} \cdots t_{n}^{a_{m n}-a_{1 n}}\right)\right] .}
\end{gathered}
$$

By taking $b_{i j}=a_{i j}-a_{1 j}$ for all $i=2, \ldots, m, j=1, \ldots, n$, we obtain

$$
X=\left\{\left[\left(1, t_{1}^{b_{21}} \cdots t_{n}^{b_{2 n}}, \ldots, t_{1}^{b_{m 1}} \cdots t_{n}^{b_{m n}}\right)\right] \in \mathbb{P}^{m-1}: t_{i} \in K^{*}\right\} .
$$

From now on we will use any of the representations (1) or (3) to mean an algebraic toric set parameterized by the monomials $Z^{a_{1}}, \ldots, Z^{a_{m}}$ or, in an equivalent way, to represent an algebraic toric set associated to the matrix (2).

Let $S=K\left[X_{1}, \ldots, X_{m}\right]=\oplus_{d=0}^{\infty} S_{d}$ be a polynomial ring over the field $K$ with the standard grading, let $\left[P_{1}\right], \ldots,\left[P_{|X|}\right]$ be the points of $X$, and let $f_{0}\left(X_{1}, \ldots, X_{m}\right)=X_{1}^{d}$. The evaluation map

$$
\begin{aligned}
\mathrm{ev}_{d}: S_{d} & =K\left[X_{1}, \ldots, X_{m}\right]_{d} \rightarrow K^{|X|}, \\
f & \mapsto\left(\frac{f\left(P_{1}\right)}{f_{0}\left(P_{1}\right)}, \ldots, \frac{f\left(P_{|X|}\right)}{f_{0}\left(P_{|X|}\right)}\right)
\end{aligned}
$$

defines a linear map of $K$-vector spaces. The image of $\mathrm{ev}_{d}$, denoted by $C_{X}(d)$, defines a linear code. We will call $C_{X}(d)$ a projective parameterized code of order $d$ arising from the toric set $X$ or associated to the matrix $A$. As usual by a linear code we mean a linear subspace of $K^{|X|}$.

In this paper we will only deal with projective parameterized codes arising from the set $X$, defined in (1) or (3), over finite fields and we will describe their main characteristics. 
The dimension and length of the code $C_{X}(d)$ are given by $\operatorname{dim}_{K} C_{X}(d)$ and $|X|$ respectively. The dimension and length are two of the basic parameters of a linear code. A third basic parameter is the minimum distance which is given by

$$
\delta_{X}(d)=\min \left\{\|v\|: 0 \neq v \in C_{X}(d)\right\},
$$

where $\|v\|$ is the number of non-zero entries of $v$. The basic parameters of $C_{X}(d)$ are related by the Singleton bound which is an upper bound for the minimum distance

$$
\delta_{X}(d) \leq|X|-\operatorname{dim}_{K} C_{X}(d)+1
$$

Projective parameterized codes are important because in some cases their main parameters have the best behavior. For example in [7] the resulting codes are MDS.

The parameters of evaluation codes over finite fields have been computed in several cases. Our approximation, when we consider the evaluation codes as associated to the matrix (2), generalizes many cases studied previously. For example if $A=I_{m}$, the projective parameterized codes associated to $A$ become the Generalized Reed-Solomon codes [8]. If $X=\mathbb{P}^{m-1}$, the parameters of $C_{X}(d)$ are described in [20, Theorem 1]. If $X$ is the image of the affine space $\mathbb{A}^{m-1}$ under the map $\mathbb{A}^{m-1} \rightarrow \mathbb{P}^{m-1}, x \mapsto[(1, x)]$, the parameters of $C_{X}(d)$ are described in [2, Theorem 2.6.2]. Also if we consider the matrix (2) as the incidence matrix of a graph $G$, we obtain the projective parameterized codes associated to $G$. In the following sections when we write graph we mean a simple graph, i.e., an undirected graph that has no loops and no more than one edge between any two different vertices. The main characteristics of evaluation codes associated to complete bipartite graphs were found in [6]. Some general results over projective parameterized codes were described in [16].

It is worth saying that projective parameterized codes are, in general, strictly different to toric codes which were defined in [11] and generalized for example in [13] and [19]. They evaluate over the complete torus, meanwhile we do it over specific subsets of the projective space.

In this work we will analyze the case where the parameterized codes of order $d, C_{X}(d)$, come from the general matrix (2) and we will estimate their main parameters.

The vanishing ideal of $X$, denoted by $I_{X}$, is the ideal of $S$ generated by the homogeneous polynomials of $S$ that vanish on $X$.

For all unexplained terminology and additional information we refer to [1, 21] (for the theory of polynomial ideals and Hilbert functions), and [14, 22, 24] (for the theory of error-correcting codes and algebraic geometric codes). 


\section{Preliminaries}

In the following we will use the notation and definitions given in the introduction. In this section we introduce the basic algebraic invariants of $S / I_{X}$ and their connection with the basic parameters of projective parameterized linear codes. Then we present some of the results that we are going to use later.

Recall that the projective space of dimension $m-1$ over $K$, denoted by $\mathbb{P}^{m-1}$, is the quotient space

$$
\left(K^{m} \backslash\{0\}\right) / \sim
$$

where two points $\alpha, \beta$ in $K^{m} \backslash\{0\}$ are equivalent if $\alpha=\lambda \beta$ for some $\lambda \in K$. We denote the equivalence class of $\alpha$ by $[\alpha]$. Let $X \subset \mathbb{P}^{m-1}$ be an algebraic toric set parameterized by $Z^{a_{1}}, \ldots, Z^{a_{m}}$ and let $C_{X}(d)$ be a projective parameterized code of order $d$. The kernel of the evaluation map ev ${ }_{d}$, defined in (4), is precisely $I_{X}(d)$, the degree $d$ piece of $I_{X}$. Therefore there is an isomorphism of $K$-vector spaces

$$
S_{d} / I_{X}(d) \simeq C_{X}(d) .
$$

Two of the basic parameters of $C_{X}(d)$ can be expressed using Hilbert functions of standard graded algebras [21], as we now explain. Recall that the Hilbert function of $S / I_{X}$ is given by

$$
H_{X}(d)=\operatorname{dim}_{K}\left(S / I_{X}\right)_{d}=\operatorname{dim}_{K} S_{d} / I_{X}(d)=\operatorname{dim}_{K} C_{X}(d) .
$$

The unique polynomial $h_{X}(t)=\sum_{i=0}^{k-1} c_{i} t^{i} \in \mathbb{Z}[t]$ of degree $k-1=$ $\operatorname{dim}\left(S / I_{X}\right)-1$ such that $h_{X}(d)=H_{X}(d)$ for $d \gg 0$ is called the Hilbert polynomial of $S / I_{X}$. The integer $c_{k-1}(k-1)$ !, denoted by $\operatorname{deg}\left(S / I_{X}\right)$, is called the degree or multiplicity of $S / I_{X}$. In our situation $h_{X}(t)$ is a non-zero constant because $S / I_{X}$ has dimension 1 . Furthermore $h_{X}(d)=|X|$ for $d \geq|X|-1$, see [12, Lecture 13]. This means that $|X|$ equals the degree of $S / I_{X}$. Thus $H_{X}(d)$ and $\operatorname{deg}\left(S / I_{X}\right)$ equal the dimension and the length of $C_{X}(d)$ respectively. There are algebraic methods, based on elimination theory and Gröbner bases, to compute the dimension and the length of $C_{X}(d)[16]$.

The regularity index of $S / I_{X}$, denoted by $\operatorname{reg}\left(S / I_{X}\right)$, is the least integer $p \geq 0$ such that $h_{X}(d)=H_{X}(d)$ for $d \geq p$. The degree and the regularity index can be read off the Hilbert series as we now explain. The Hilbert series of $S / I_{X}$ can be written as

$$
F_{X}(t)=\sum_{i=0}^{\infty} H_{X}(i) t^{i}=\sum_{i=0}^{\infty} \operatorname{dim}_{K}\left(S / I_{X}\right)_{i} t^{i}=\frac{h_{0}+h_{1} t+\cdots+h_{r} t^{r}}{1-t},
$$

where $h_{0}, \ldots, h_{r}$ are positive integers. In fact we have that

$$
h_{i}=\operatorname{dim}_{K}\left(S /\left(I_{X}, X_{m}\right)\right)_{i}
$$


for $0 \leq i \leq r$ and $\operatorname{dim}_{K}\left(S /\left(I_{X}, X_{m}\right)\right)_{i}=0$ for $i>r$. This follows from the fact that $I_{X}$ is a Cohen-Macaulay lattice ideal [16] and by observing that $\left\{X_{m}\right\}$ is a regular system of parameters for $S / I_{X}$ (see [21]). The number $r$ equals the regularity index of $S / I_{X}$ and the degree of $S / I_{X}$ equals $h_{0}+\cdots+h_{r}$ (see [21] or [25, Corollary 4.1.12]).

The regularity index plays a very important role in the study of evaluation codes arising from a set $X$ because in the case $d \geq \operatorname{reg}\left(S / I_{X}\right)$ we obtain that $H_{X}(d)=|X|$ and then $C_{X}(d)=K^{|X|}$, which is a trivial case. Therefore we always work with $0 \leq d<\operatorname{reg}\left(S / I_{X}\right)$. Another motivation to study the regularity index comes from commutative algebra because, in this case, $\operatorname{reg}\left(S / I_{X}\right)$ is equal to the Castelnuovo-Mumford regularity which is an algebraic invariant of central importance [4].

\section{Main Results}

From now on we will work with the toric set $X$ defined in (1) or (3) and our goal is to describe the main parameters of the projective parameterized codes of order $d, C_{X}(d)$, which were defined as the image of the evaluation map $\mathrm{ev}_{d}$ introduced in (4).

\subsection{Length}

In order to cumpute the length of the projective parameterized codes arising from the toric set $X$, we introduce the following multiplicative subgroups of $X$ for all $i=1, \ldots, n$.

$$
Y_{i}:=\left\{\left[\left(1, t_{i}^{b_{2 i}}, \ldots, t_{i}^{b_{m i}}\right)\right] \in \mathbb{P}^{m-1}: t_{i} \in K^{*} \text { for all } i\right\}
$$

It is easy to see that $\left|Y_{i}\right|=\frac{q-1}{\left(q-1, b_{2 i}, \ldots, b_{m i}\right)}$ for all $i=1, \ldots, n$, where $\left(q-1, b_{2 i}, \ldots, b_{m i}\right)$ means the greatest common divisor of the corresponding integers. With this information we are able to prove the main result of this section.

Theorem 3.1. The length of the projective parameterized codes of order $d$, $C_{X}(d)$, is given by

$$
|X|=\frac{1}{|M|} \prod_{i=1}^{n}\left|Y_{i}\right|
$$

where $M$ is the set of $n$-tuples $\left(i_{1}, \ldots, i_{n}\right)$ such that

$$
1 \leq i_{j} \leq \frac{q-1}{\left(q-1, b_{2 j}, \ldots, b_{m j}\right)} \quad \text { for all } j=1, \ldots, n
$$


and

$$
\begin{gathered}
i_{1} b_{21}+i_{2} b_{22}+\cdots+i_{n} b_{2 n} \equiv 0 \bmod (q-1) \\
i_{1} b_{31}+i_{2} b_{32}+\cdots+i_{n} b_{3 n} \equiv 0 \bmod (q-1) \\
\cdots \cdots \cdots \cdots \cdots \cdots \cdots \cdots \cdots \cdots \cdots \cdots \cdots \cdots \\
i_{1} b_{m 1}+i_{2} b_{m 2}+\cdots+i_{n} b_{m n} \equiv 0 \bmod (q-1)
\end{gathered}
$$

Proof. Let $\phi$ be the following map

$$
\begin{gathered}
\phi: Y_{1} \times \cdots \times Y_{n} \rightarrow X, \\
\phi\left(\left[\left(1, t_{1}^{b_{21}}, \ldots, t_{1}^{b_{m 1}}\right)\right], \ldots,\left[\left(1, t_{n}^{b_{2 n}}, \ldots, t_{n}^{b_{m n}}\right)\right]\right)= \\
{\left[\left(1, t_{1}^{b_{21}} \cdots t_{n}^{b_{2 n}}, \ldots, t_{1}^{b_{m 1}} \cdots t_{n}^{b_{m n}}\right)\right] .}
\end{gathered}
$$

It is immediate that $\phi$ is an epimorphism between multiplicative groups. Thus

$$
|X|=\frac{\left|Y_{1} \times \cdots \times Y_{n}\right|}{|\operatorname{ker} \phi|}=\frac{1}{|\operatorname{ker} \phi|} \prod_{i=1}^{n}\left|Y_{i}\right| .
$$

Let $\beta$ a generator of $\left(K^{*}, \cdot\right)$. Therefore

$$
\begin{aligned}
\operatorname{ker} \phi=\{ & \left(\left[\left(1, \beta^{i_{1} b_{21}}, \ldots, \beta^{i_{1} b_{m 1}}\right)\right], \ldots,\left[\left(\beta^{i_{n} b_{2 n}}, \ldots, \beta^{i_{n} b_{m n}}\right)\right]\right) \in Y_{1} \times \cdots \times Y_{n}: \\
& {\left.\left[\left(1, \beta^{i_{1} b_{21}+\cdots+i_{n} b_{2 n}}, \ldots, \beta^{i_{1} b_{m 1}+\cdots+i_{n} b_{m n}}\right)\right]=[(1,1, \ldots, 1)]\right\} . }
\end{aligned}
$$

In this case $\beta^{i_{1} b_{21}+\cdots+i_{n} b_{2 n}}=1, \ldots, \beta^{i_{1} b_{m 1}+\cdots+i_{n} b_{m n}}=1$. These equalities imply the system of congruences (6). Then there is a bijection between ker $\phi$ and the set of $n$-tuples $\left(i_{1}, \ldots, i_{n}\right)$ such that $1 \leq i_{j} \leq\left|Y_{j}\right|$ for all $j=1, \ldots, n$ and satisfy (6).

The equation (5) follows immediately from last results.

We define the projective torus of dimension $m-1$ as

$$
\mathbb{T}_{m-1}=\left\{\left[\left(c_{1}, \ldots, c_{m}\right)\right] \in \mathbb{P}^{m-1}: c_{i} \in K^{*} \text { for all } i\right\} .
$$

Obviously, $X \subseteq \mathbb{T}_{m-1}$. The following corollary is an easy consequence of Theorem 3.1. It gives the conditions under which last inclusion becomes an equality.

Corollary 3.2. If $n=m$ then $X$ is the projective torus of dimension $m-1$ if and only if $|M|=1$ and $\left(q-1, b_{2 j}, \ldots, b_{m j}\right)=1$ for all $j=1, \ldots, n$.

On the other hand if we consider the case where the monomials that parameterize the toric set $X$ are all of them of the same degree then we obtain another corollary. 
Corollary 3.3. If the sum of the elements of each column of the matrix $A$ defined in (2) is a constant or, equivalently, the monomials that parameterize the toric set $X$ are all of them of the same degree, then $|X| \leq(q-1)^{n-1}$.

Proof. Let $\sum_{j=1}^{n} a_{i j}=\alpha$ (a positive integer) for all $i=1, \ldots, m$. We note that $\left|Y_{i}\right| \leq q-1$ for all $i=1, \ldots, n$. Moreover

$$
\sum_{j=1}^{n} b_{i j}=\sum_{j=1}^{n} a_{i j}-\sum_{j=1}^{n} a_{1 j}=\alpha-\alpha=0,
$$

and then $(1, \ldots, 1) \in M$. Let $\gamma=\min \left\{\left|Y_{i}\right|: i=1, \ldots, n\right\}$. Therefore $(j, \ldots, j) \in M$ for all $1 \leq j \leq \gamma$ and it implies that $|M| \geq \gamma$. Thus

$$
|X|=\frac{1}{|M|} \prod_{i=1}^{n}\left|Y_{i}\right| \leq \frac{\gamma(q-1)^{n-1}}{\gamma}=(q-1)^{n-1}
$$

and the claim follows.

Remark 3.4. If $G$ is a graph and $X$ is the algebraic toric set associated to the incidence matrix of $G$, then the sum of the elements of each column of this matrix is $\alpha=2$ and the result of the last corollary follows. Actually in this situation $\left|Y_{i}\right|=q-1$ for all $i=1, \ldots, n$ and then we get that in any graph $|X|=\frac{(q-1)^{n}}{|M|}$. Moreover in [16, Corollary 3.8] it was found the exact value of $|X|$ if $G$ is a connected graph. By using this result we obtain that

$$
|M|= \begin{cases}(q-1)^{2} & \text { if } \quad G \text { is bipartite } \\ q-1 & \text { if } \quad G \text { is non-bipartite. }\end{cases}
$$

On the other hand if we consider disconnected graphs, in [15, Theorem 3.2] it was found the exact value of $|X|$, which is a generalization of [16, Corollary 3.8].

\subsection{Dimension}

In the following theorem we give the dimension of the projective parameterized codes arising from the algebraic toric set $X$ in terms of the dimension of the projective parameterized codes arising from the projective torus $\mathbb{T}_{m-1}$, which is well known (see [8]).

Theorem 3.5. The dimension of the projective parameterized codes of order $d, C_{X}(d)$, is given by

$$
H_{X}(d)=H_{\mathbb{T}_{m}-1}(d)-\bar{H}(d)
$$

for all $d \geq 0$ and where $\bar{H}$ is the Hilbert function of $I_{X} / I_{\mathbb{T}_{m-1}}$, i.e.,

$$
\bar{H}(d)=\operatorname{dim}_{K} I_{X}(d) / I_{\mathbb{T}_{m-1}}(d) .
$$


Proof. We know that $X \subseteq \mathbb{T}_{m-1}$ and then $I_{\mathbb{T}_{m-1}} \subseteq I_{X}$. Let $\psi$ be the following linear transformation.

$$
\begin{aligned}
\psi: S_{d} / I_{\mathbb{T}_{m-1}}(d) & \rightarrow S_{d} / I_{X}(d), \\
f+I_{\mathbb{T}_{m-1}}(d) & \rightarrow f+I_{X}(d) .
\end{aligned}
$$

This a well defined function and in fact it is a surjective linear map. Moreover $\operatorname{ker} \psi=I_{X}(d) / I_{\mathbb{T}_{m-1}}(d)$. Thus

$$
\begin{gathered}
\operatorname{dim}_{K} S_{d} / I_{\mathbb{T}_{m-1}}(d)= \\
\operatorname{dim}_{K} S_{d} / I_{X}(d)+\operatorname{dim}_{K} I_{X}(d) / I_{\mathbb{T}_{m-1}}(d),
\end{gathered}
$$

and the equality (8) follows immediately.

For the following corollary we will use $r_{X}, r_{\mathbb{T}_{m-1}}$ and $r_{\bar{H}}$ as the regularity indexes of $S / I_{X}, S / I_{\mathbb{T}_{m-1}}$ and $I_{X} / I_{\mathbb{T}_{m-1}}$, respectively.

Corollary 3.6. $r_{\mathbb{T}_{m-1}}=\max \left\{r_{X}, r_{\bar{H}}\right\}$.

Proof. Let

$$
\begin{gathered}
\theta: I_{X}(d) / I_{\mathbb{T}_{m-1}}(d) \rightarrow I_{X}(d+1) / I_{\mathbb{T}_{m-1}}(d+1), \\
\theta\left(f+I_{\mathbb{T}_{m-1}}(d)\right)=X_{1} f+I_{\mathbb{T}_{m-1}}(d+1) .
\end{gathered}
$$

It is easy to see that $\theta$ is a well defined map, moreover it is a linear transformation. If $f+I_{\mathbb{T}_{m-1}}(d) \in \operatorname{ker} \theta$ then $X_{1} f \in I_{\mathbb{T}_{m-1}}(d+1)$. Let $[P]=$ $\left[\left(1, t_{1}^{b_{21}} \cdots t_{n}^{b_{2 n}}, \ldots, t_{1}^{b_{m 1}} \cdots t_{n}^{b_{m n}}\right)\right] \in X$. Thus $\left(X_{1} f\right)(P)=0$ and then $f(P)=$ 0 . Therefore $f \in I_{\mathbb{T}_{m-1}}(d)$ and $\operatorname{ker} \theta=I_{\mathbb{T}_{m-1}}(d)$. It implies that $\bar{H}(d) \leq$ $\bar{H}(d+1)$ for all $d \geq 0$. By the last inequality and (8) the claim follows.

Remark 3.7. By the Corollary 3.6 we obtain that $r_{X} \leq r_{\mathbb{T}_{m-1}}$. But in [8] it was proved that $r_{\mathbb{T}_{m-1}}=(m-1)(q-2)$. Therefore

$$
r_{X} \leq(m-1)(q-2) .
$$

As we observed in section 2, if $d \geq(m-1)(q-2)$ then $H_{X}(d)=|X|$ and thus $C_{X}(d)=K^{|X|}$. Therefore from now on we will use $d<(m-1)(q-2)$. 


\subsection{Minimum distance}

The minimum distance has been computed in several cases associated to evaluation codes. In particular in [18] it was computed when we consider projective parameterized codes arising from the projective torus. Moreover in [23] some lower bounds on the minimum distance were found coming from syzigies. In this section we are going to find an upper bound for the minimum distance of any projective parameterized code and we will find a lower bound for this kind of codes when the sum of the elements of each column of the matrix (2) becomes a constant. We consider that $X \subset \mathbb{T}_{m-1}$ because the case $X=\mathbb{T}_{m-1}$ is well known (see [8]). Let $Y:=\mathbb{T}_{m-1} \backslash X$ and $\delta_{X}(d), \delta_{Y}(d)$ and $\delta_{\mathbb{T}_{m-1}}(d)$ be the minimum distances of the parameterized codes of order $d, C_{X}(d), C_{Y}(d)$ and $C_{\mathbb{T}_{m-1}}(d)$, respectively. The following theorem relates them.

Theorem 3.8. Let $0 \leq d<(m-1)(q-2)$. Then

$$
\delta_{X}(d) \leq \delta_{\mathbb{T}_{m-1}}(d)-\delta_{Y}(d) .
$$

Proof. Let $X=\left\{\left[P_{1}\right], \ldots,\left[P_{|X|}\right]\right\}$. We can write

$$
\mathbb{T}_{m-1}=\left\{\left[P_{1}\right], \ldots,\left[P_{|X|}\right],\left[Q_{1}\right], \ldots,\left[Q_{|Y|}\right]\right\},
$$

where of course $Y=\left\{\left[Q_{1}\right], \ldots,\left[Q_{|Y|}\right]\right\}$. If

$$
\Lambda=\left(\frac{f\left(P_{1}\right)}{X_{1}^{d}\left(P_{1}\right)}, \ldots, \frac{f\left(P_{|X|}\right)}{X_{1}^{d}\left(P_{|X|}\right)}, \frac{f\left(Q_{1}\right)}{X_{1}^{d}\left(Q_{1}\right)}, \ldots, \frac{f\left(Q_{|Y|}\right)}{X_{1}^{d}\left(Q_{|Y|}\right)}\right) \in C_{\mathbb{T}_{m-1}}(d)
$$

with $w(\Lambda)=\delta_{\mathbb{T}_{m-1}}(d)$, where $w(\Lambda)$ is the Hamming weight of the codeword $\Lambda$, then

$$
\Lambda_{1}:=\left(\frac{f\left(P_{1}\right)}{X_{1}^{d}\left(P_{1}\right)}, \ldots, \frac{f\left(P_{|X|}\right)}{X_{1}^{d}\left(P_{|X|}\right)}\right) \in C_{X}(d),
$$

and

$$
\Lambda_{2}:=\left(\frac{f\left(Q_{1}\right)}{X_{1}^{d}\left(Q_{1}\right)}, \ldots, \frac{f\left(Q_{|Y|}\right)}{X_{1}^{d}\left(Q_{|Y|}\right)}\right) \in C_{Y}(d) .
$$

Moreover

$$
\delta_{\mathbb{T}_{m-1}}(d)=w(\Lambda)=w\left(\Lambda_{1}\right)+w\left(\Lambda_{2}\right) \geq \delta_{X}(d)+\delta_{Y}(d) .
$$

Therefore the inequality (11) follows from (12).

Remark 3.9. From the inequality (11) we obtain that $\delta_{X}(d) \leq \delta_{\mathbb{T}_{m}-1}(d)-1$ for all $0 \leq d<(m-1)(q-2)$. But $\delta_{\mathbb{T}_{m-1}}(d)$ was computed in [18]. Thus in this case

$$
\delta_{X}(d) \leq(q-1)^{m-(k+2)}(q-1-\ell)-1,
$$

where $k$ and $\ell$ are the unique integers such that $k \geq 0,1 \leq \ell \leq q-2$ and $d=k(q-2)+\ell$. 
From now on we will consider the case worked in section 3.1, where the sum of the elements of each column of the matrix $A$ defined in (2) is a constant, i.e., $\sum_{j=1}^{n} a_{i j}=\alpha$ (a positive integer) for all $i=1, \ldots, m$. The following map will help us to find a lower bound for the minimum distance of the corresponding projective parameterized codes.

$$
\begin{gathered}
\mu: \mathbb{T}_{n-1} \rightarrow X \\
{\left[\left(t_{1}, \ldots, t_{n}\right)\right] \rightarrow\left[\left(t_{1}^{a_{11}} \cdots t_{n}^{a_{1 n}}, \ldots, t_{1}^{a_{m 1}} \cdots t_{n}^{a_{m n}}\right)\right] .}
\end{gathered}
$$

$\mu$ is a well defined map and in fact it is an epimorphism of multiplicative groups. Let $N:=\operatorname{ker} \mu$. Thus $|N|=\frac{\left|\mathbb{T}_{n-1}\right|}{|X|}=\frac{(q-1)^{n-1}}{|X|}$. Moreover $\mathbb{T}_{n-1}=$ $\cup_{i=1}^{|X|} N \cdot\left[Q_{i}\right]$ (disjoint union of the corresponding cosets) for some $\left[Q_{i}\right] \in \mathbb{T}_{n-1}$. Let $\left[P_{i}\right]=\mu\left(\left[Q_{i}\right]\right)$ for all $i=1, \ldots,|X|$ and $N=\left\{\left[R_{1}\right], \ldots,\left[R_{|N|}\right]\right\}$. Thus $X=\left\{\left[P_{1}\right], \ldots,\left[P_{|X|}\right]\right\}$ and

$$
\mathbb{T}_{n-1}=\left\{\left[R_{1} Q_{1}\right], \ldots,\left[R_{|N|} Q_{1}\right], \ldots,\left[R_{1} Q_{|X|}\right], \ldots,\left[R_{|N|} Q_{|X|}\right]\right\} .
$$

As in the introduction let $L=K\left[Z_{1}, \ldots, Z_{n}\right]$. We define another map that will be useful later on.

$$
\begin{gathered}
\tau: S_{d} \rightarrow L_{\alpha d}, \\
f\left(X_{1}, \ldots, X_{m}\right) \rightarrow f\left(Z_{1}^{a_{11}} \cdots Z_{n}^{a_{1 n}}, \ldots, Z_{1}^{a_{m 1}} \cdots Z_{n}^{a_{m n}}\right) .
\end{gathered}
$$

$\tau$ is a linear map between the vector spaces $S_{d}$ and $L_{\alpha d}$. Now we are able to prove the following theorem. In this result we are going to find a lower bound for the minimum distance of the corresponding projective parameterized codes.

Theorem 3.10. If the sum of the elements of each column of the matrix $A$ defined in (2) is a constant $\alpha$, then

$$
\delta_{X}(d) \geq\left\lceil\frac{|X| \cdot \delta_{\mathbb{T}_{n-1}}(\alpha d)}{(q-1)^{n-1}}\right\rceil
$$

where $\delta_{\mathbb{T}_{n-1}}(\alpha d)$ is the minimum distance of the parametererized code of order $\alpha d$ arising from the projective torus $\mathbb{T}_{n-1}$ and $\delta_{X}(d)$ is the minimum distance of the projective parameterized code associated to the toric set $X$ defined in (1). Also $\lceil x\rceil$ is the ceiling function of $x$, and it means that $\lceil x\rceil=\min \{y \in$ $\mathbb{Z}: y \geq x\}$.

Proof. Let

$$
\Gamma=\left(\frac{f\left(P_{1}\right)}{X_{1}^{d}\left(P_{1}\right)}, \ldots, \frac{f\left(P_{|X|}\right)}{X_{1}^{d}\left(P_{|X|}\right)}\right) \in C_{X}(d)
$$


We choose $\Gamma$ in such a way that $w(\Gamma)=\delta_{X}(d)$. On the other hand let

$$
\begin{gathered}
\Omega=\left(\frac{\tau(f)\left(R_{1} Q_{1}\right)}{Z_{1}^{\alpha d}\left(R_{1} Q_{1}\right)}, \ldots, \frac{\tau(f)\left(R_{|N|} Q_{1}\right)}{Z_{1}^{\alpha d}\left(R_{|N|} Q_{1}\right)}, \ldots, \frac{\tau(f)\left(R_{1} Q_{|X|}\right)}{Z_{1}^{\alpha d}\left(R_{1} Q_{|X|}\right)}, \ldots,\right. \\
\left.\frac{\tau(f)\left(R_{|N|} Q_{|X|}\right)}{Z_{1}^{\alpha d}\left(R_{|N|} Q_{|X|}\right)}\right) .
\end{gathered}
$$

We have that $\Omega \in C_{\mathbb{T}_{n-1}}(\alpha d)$ and if $f\left(P_{i}\right) \neq 0$ for some $\left[P_{i}\right] \in X$, then due to the fact that $\mu\left(\left[R_{j} Q_{i}\right]\right)=\left[P_{i}\right]$, we obtain that $\tau(f)\left(R_{j} Q_{i}\right)=f\left(P_{i}\right) \neq 0$ for all $j=1, \ldots, n$. Thus $w(\Omega)=|N| \cdot w(\Gamma)=|N| \cdot \delta_{X}(d)$ and thererefore $\delta_{\mathbb{T}_{n-1}}(\alpha d) \leq w(\Omega)=|N| \cdot \delta_{X}(d)$. Then

$$
\delta_{X}(d) \geq \frac{\delta_{\mathbb{T}_{n-1}}(\alpha d)}{|N|} .
$$

The inequality (14) follows from (15) and the fact that $|N|=\frac{(q-1)^{n-1}}{|X|}$.

If $X$ is the algebraic toric set arising from the incidence matrix of any graph then $\alpha=2$ and we can apply Theorem 3.10. Moreover if we have a connected graph, by using [16, Corollary 3.8$]$ we obtain the following general result.

Corollary 3.11. Let $X$ be the algebraic toric set arising from the incidence matrix of any connected graph $G$. Then

$$
\delta_{X}(d) \geq \begin{cases}\left\lceil\frac{\delta_{\mathbb{T}_{n-1}}(2 d)}{q-1}\right\rceil & \text { if } \quad G \text { is bipartite } \\ \delta_{\mathbb{T}_{n-1}}(2 d) & \text { if } \quad G \text { is non-bipartite. }\end{cases}
$$

Corollary 3.12. If the sum of the elements of each column of the matrix $A$ defined in (2) is a constant $\alpha$, then

$$
r_{X} \geq\left\lceil\frac{|X|(q-2)(n-1)}{\alpha(q-1)^{n-1}}\right\rceil,
$$

where $r_{X}$ is the regularity index of $S / I_{X}$.

Moreover if $G$ is a connected graph and $X$ is the algebraic toric set arising from its incidence matrix, then

$$
r_{X} \geq \begin{cases}\left\lceil\frac{(q-2)(n-1)}{2(q-1)}\right\rceil & \text { if } \quad G \text { is bipartite } \\ \left\lceil\frac{(q-2)(n-1)}{2}\right\rceil & \text { if } \quad G \text { is non-bipartite. }\end{cases}
$$




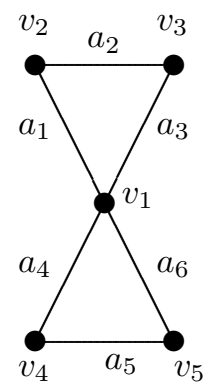

Figure 1: A connected non-bipartite graph with two cycles of length 3.

Proof. The claim follows directly because of (14), (16), and the fact that the regularity index corresponding to the torus $\mathbb{T}_{n-1}$ is exactly $(q-2)(n-1)$.

In the first example of the following section we will realize that this lower bound is attained in some cases.

\section{Examples}

In this section we will give three different examples. In the first example we will consider a particular connected non-bipartite graph and we will compute the main characteristics of the corresponding projective parameterized codes arising from the incidence matrix of that graph. In the second example we will define clutters as particular cases of hypergraphs and a specific example of projective parameterized codes arising from uniform clutters will be given. Finally in the third example we will compute the main parameters of the projective parameterized codes associated to a matrix that does not represent a clutter and then it does not represent a graph. In these examples we will use the notation appeared in the previous sections and we will use Macaulay2 [10] for the main computations. Also we will use $\delta_{d}^{\prime}$ to represent the lower bound showed in (14) and $b_{d}$ will represent the Singleton bound, i.e.,

$$
\delta_{d}^{\prime}=\left\lceil\frac{|X| \cdot \delta_{\mathbb{T}_{n-1}}(\alpha d)}{(q-1)^{n-1}}\right\rceil \text { and } b_{d}=|X|-H_{X}(d)+1 .
$$

\subsection{Example 1}

Let $G$ be the graph given in Fig. 1 where $V(G)=\left\{v_{1}, v_{2}, v_{3}, v_{4}, v_{5}\right\}$ is its vertex set and its edge set is given by $E(G)=\left\{a_{1}, a_{2}, a_{3}, a_{4}, a_{5}, a_{6}\right\}$. The 
incidence matrix of $G$ is the $5 \times 6$ matrix given by

$$
A=\left(\begin{array}{llllll}
1 & 0 & 1 & 1 & 0 & 1 \\
1 & 1 & 0 & 0 & 0 & 0 \\
0 & 1 & 1 & 0 & 0 & 0 \\
0 & 0 & 0 & 1 & 1 & 0 \\
0 & 0 & 0 & 0 & 1 & 1
\end{array}\right)
$$

Let $K=\mathbb{F}_{7}$ be a finite field with 7 elements. The toric set arising from the matrix (19) (or associated to the graph $G$ showed in Fig. 1) is given by

$$
X=\left\{\left[\left(t_{1} t_{2}, t_{2} t_{3}, t_{1} t_{3}, t_{1} t_{4}, t_{4} t_{5}, t_{1} t_{5}\right)\right] \in \mathbb{P}^{5}: t_{i} \in K^{*}\right\} .
$$

In this case we have five subsets $Y_{i}$ with $\left|Y_{i}\right|=6$ for all $i=1, \ldots, 5$. The corresponding subset $M$ is

$$
M=\{(i, i, i, i, i): i=1, \ldots, 6\},
$$

and therefore, by using Theorem 3.1,

$$
|X|=\frac{1}{|M|} \prod_{i=1}^{5}\left|Y_{i}\right|=1296 .
$$

We notice that $\delta_{d}^{\prime}=\delta_{\mathbb{T}_{4}}(2 d)$ because of Corollary 3.11. By using Macaulay2 we compute the following values.

\begin{tabular}{||c||c||c||c||c||c||}
\hline$d$ & 1 & 2 & 3 & 4 & 5 \\
\hline$H_{X}(d)$ & 6 & 21 & 55 & 120 & 231 \\
\hline$H_{\mathbb{T}_{5}}(d)$ & 6 & 21 & 56 & 126 & 252 \\
\hline $\bar{H}(d)$ & 0 & 0 & 1 & 6 & 21 \\
\hline$\delta_{d}^{\prime}$ & 864 & 432 & 180 & 108 & 36 \\
\hline$b_{d}$ & 1291 & 1276 & 1242 & 1177 & 1066 \\
\hline
\end{tabular}

\begin{tabular}{||c||c||c||c||c||c||}
\hline$d$ & 6 & 7 & 8 & 9 & 10 \\
\hline$H_{X}(d)$ & 401 & 627 & 885 & 1130 & 1296 \\
\hline$H_{\mathbb{T}_{5}}(d)$ & 457 & 762 & 1182 & 1722 & 2373 \\
\hline $\bar{H}(d)$ & 56 & 135 & 297 & 592 & 1077 \\
\hline$\delta_{d}^{\prime}$ & 24 & 12 & 5 & 3 & 1 \\
\hline$b_{d}$ & 896 & 670 & 412 & 167 & 1 \\
\hline
\end{tabular}

Moreover in this case the regularity index is $r_{X}=10=\frac{(q-2)(n-1)}{2}$ and it shows that the lower bound given in (18) works very well. This lower bound is attained in this particular case. 


\subsection{Example 2}

In this example we continue using the notation used in the introduction.

A clutter $\mathcal{C}$ is a family $E$ of subsets of a finite ground set $Z=\left\{Z_{1}, \ldots, Z_{n}\right\}$ such that if $h_{1}, h_{2} \in E$, then $h_{1} \not \subset h_{2}$. The ground set $Z$ is called the vertex set of $\mathcal{C}$ and $E$ is called the edge set of $\mathcal{C}$ and they are denoted by $V_{\mathcal{C}}$ and $E_{\mathcal{C}}$ respectively.

Clutters are special hypergraphs and are sometimes called Sperner families in the literature. One example of a Clutter is a graph with the vertices and edges defined in the usual way for graphs.

Let $\mathcal{C}$ be a clutter with vertex set $V_{\mathcal{C}}=\left\{Z_{1}, \ldots, Z_{n}\right\}$ and let $h$ be an edge

of $\mathcal{C}$. The characteristic vector of $h$ is the vector $a=\sum_{Z_{i} \in h} e_{i}$ where $e_{i}$ is the $i t h$ unit vector in $\mathbb{R}^{n}$. Throughout this example we assume that $a_{1}, \ldots, a_{m}$ is the set of all characteristic vectors of the edges of $\mathcal{C}$. In this case the matrix (2) is known as the incidence matrix of the clutter $\mathcal{C}$ and the set $X$ defined in (1) is the toric set associated to the clutter $\mathcal{C}$. The clutter $\mathcal{C}$ is called uniform if the sum of the elements of the columns of its incidence matrix is a constant.

We realize that in any clutter, like in graphs, $|X|=\frac{(q-1)^{n}}{|M|}$ because $\left|Y_{i}\right|=$ $q-1$ for all $i=1, \ldots, n$.

Let $K=\mathbb{F}_{9}$ be a finite field with 9 elements and $X$ be the toric set associated to the uniform clutter $(\alpha=3)$ whose incidence matrix is the $6 \times 6$ matrix given by

$$
A=\left(\begin{array}{llllll}
1 & 0 & 0 & 0 & 1 & 1 \\
1 & 1 & 0 & 0 & 0 & 1 \\
1 & 1 & 1 & 0 & 0 & 0 \\
0 & 1 & 1 & 1 & 0 & 0 \\
0 & 0 & 1 & 1 & 1 & 0 \\
0 & 0 & 0 & 1 & 1 & 1
\end{array}\right)
$$

The toric set $X$ associated to (20) becomes

$$
X=\left\{\left[\left(t_{1} t_{2} t_{3}, t_{2} t_{3} t_{4}, t_{3} t_{4} t_{5}, t_{4} t_{5} t_{6}, t_{1} t_{5} t_{6}, t_{1} t_{2} t_{6}\right)\right] \in \mathbb{P}^{5}: t_{i} \in K^{*}\right\} .
$$

In this case we have six subsets $Y_{i}$ with $\left|Y_{i}\right|=8$ for $i=1, \ldots, 6$. The corresponding set $M$ used in Theorem 3.1 has 512 elements and therefore, by $(5)$,

$$
|X|=\frac{1}{|M|} \prod_{i=1}^{6}\left|Y_{i}\right|=512 .
$$

In the same way that in the last example we obtain, by using Macaulay2, the following values. 


\begin{tabular}{||c||c||c||c||c||c||c||}
\hline$d$ & 1 & 2 & 3 & 4 & 5 & 6 \\
\hline$H_{X}(d)$ & 6 & 19 & 44 & 85 & 146 & 231 \\
\hline$H_{\mathbb{T}_{5}}(d)$ & 6 & 21 & 56 & 126 & 252 & 462 \\
\hline $\bar{H}(d)$ & 0 & 2 & 12 & 41 & 106 & 231 \\
\hline$\delta_{d}^{\prime}$ & 320 & 128 & 48 & 24 & 7 & 4 \\
\hline$b_{d}$ & 507 & 494 & 469 & 428 & 367 & 282 \\
\hline
\end{tabular}

\begin{tabular}{||c||c||c||c||c||c||c||}
\hline$d$ & 7 & 8 & 9 & 10 & 11 & 12 \\
\hline$H_{X}(d)$ & 344 & 442 & 492 & 510 & 512 & 512 \\
\hline$H_{\mathbb{T}_{5}}(d)$ & 792 & 1282 & 1972 & 2898 & 4088 & 5558 \\
\hline $\bar{H}(d)$ & 448 & 840 & 1480 & 2388 & 3576 & 5046 \\
\hline$\delta_{d}^{\prime}$ & 1 & 1 & 1 & 1 & 1 & 1 \\
\hline$b_{d}$ & 169 & 71 & 21 & 3 & 1 & 1 \\
\hline
\end{tabular}

It is immediate from the last table that $r_{X}=11$.

\subsection{Example 3}

In this example we will give the main characteristics of the projective parameterized codes arising from a matrix that does not represent a clutter.

Let $K=\mathbb{F}_{11}$ be a finite field with 11 elements and $X$ be the toric set associated to the $3 \times 4$ matrix given by

$$
A=\left(\begin{array}{llll}
3 & 1 & 0 & 1 \\
0 & 4 & 2 & 2 \\
3 & 1 & 4 & 3
\end{array}\right)
$$

In this case $\alpha=6$ and the set $X$ becomes

$$
X=\left\{\left[\left(t_{1}^{3} t_{3}^{3}, t_{1} t_{2}^{4} t_{3}, t_{2}^{2} t_{3}^{4}, t_{1} t_{2}^{2} t_{3}^{3}\right)\right] \in \mathbb{P}^{3}: t_{i} \in K^{*}\right\} .
$$

We have three subsets $Y_{i}$ with $\left|Y_{1}\right|=\left|Y_{3}\right|=10$ and $\left|Y_{2}\right|=5$. The corresponding subset $M$ has 10 elements and then, by Theorem 3.1,

$$
|X|=\frac{1}{|M|} \prod_{i=1}^{3}\left|Y_{i}\right|=50 .
$$

By using Macaulay2 we obtain the following values.

\begin{tabular}{||c||c||c||c||c||c||c||}
\hline$d$ & 1 & 2 & 3 & 4 & 5 & 6 \\
\hline$H_{X}(d)$ & 4 & 10 & 20 & 32 & 44 & 50 \\
\hline$H_{\mathbb{T}_{3}}(d)$ & 4 & 10 & 20 & 35 & 56 & 84 \\
\hline $\bar{H}(d)$ & 0 & 0 & 0 & 3 & 12 & 34 \\
\hline$\delta_{d}^{\prime}$ & 20 & 3 & 1 & 1 & 1 & 1 \\
\hline$b_{d}$ & 47 & 41 & 31 & 19 & 7 & 1 \\
\hline
\end{tabular}

We conclude that $r_{X}=6$. 


\section{References}

[1] W. W. Adams and P. Loustaunau, An Introduction to Gröbner Bases (GSM 3, American Mathematical Society, 1994).

[2] P. Delsarte, J. M. Goethals and F. J. MacWilliams, On generalized ReedMuller codes and their relatives, Information and Control 16 (1970) 403-442.

[3] I. M. Duursma, C. Rentería and H. Tapia-Recillas, Reed-Muller codes on complete intersections, Appl. Algebra Engrg. Comm. Comput. 11 (6) (2001) 455-462.

[4] D. Eisenbud, The geometry of syzygies: A second course in commutative algebra and algebraic geometry (Graduate Texts in Mathematics, 229, Springer-Verlag, New York, 2005).

[5] L. Gold, J. Little and H. Schenck, Cayley-Bacharach and evaluation codes on complete intersections, J. Pure Appl. Algebra 196 (1) (2005) 91-99.

[6] M. González-Sarabia and C. Rentería, Evaluation codes associated to complete bipartite graphs, Int. J. Algebra 2 (2008) 163-170.

[7] M. González-Sarabia and C. Rentería, Evaluation Codes Associated to some Matrices, Int. J. Contemp. Math. Sci. 2 (13) (2007) 615-625.

[8] M. González-Sarabia, C. Rentería and M. Hernández de la Torre, Minimum distance and second generalized Hamming weight of two particular linear codes, Congr. Numer. 161 (2003) 105-116.

[9] M. González-Sarabia, C. Rentería and H. Tapia-Recillas, Reed-Mullertype codes over the Segre variety, Finite Fields Appl. 8 (4) (2002) 511518.

[10] D. Grayson and M. Stillman, Macaulay2, Available via anonymous ftp from math.uiuc.edu, 1996.

[11] J. Hansen, Toric surfaces and error-correcting codes, Coding theory, Criptography and related areas (2000) 132-142.

[12] J. Harris, Algebraic Geometry. A first course (Graduate Texts in Mathematics 133, Springer-Verlag, New York, 1992).

[13] D. Joyner, Toric codes over finite fields, Appl. Algebra Engrg. Comm. Comput. 15 (2004) 63-79. 
[14] F.J. MacWilliams and N.J.A. Sloane, The Theory of Error-correcting Codes (North-Holland, 1977).

[15] J. Neves, M. Vaz Pinto, R.H. Villarreal, Vanishing ideals over graphs and even cycles, To appear in Comm. Algebra. (2011) arXiv:1111.6278[pdf, ps, other].

[16] C. Rentería, A. Simis and R. H. Villarreal, Algebraic methods for parameterized codes and invariants of vanishing ideals over finite fields, Finite Fields Appl.17 (2011) 81-104.

[17] E. Reyes, R. H. Villarreal and L. Zárate, A note on affine toric varieties, Linear Algebra Appl. 318 (2000) 173-179.

[18] E. Sarmiento, M. Vaz Pinto and R. H. Villarreal, The minimum distance of parameterized codes on projective tori, Appl. Algebra Engrg. Comm. Comput. 22 (4) (2011) 249-264.

[19] I. Soprunov, J. Soprunova, Bringing toric codes to the next dimension, SIAM J. Discrete Math. 24 (1) (2010) 655-665.

[20] A. Sørensen, Projective Reed-Muller codes, IEEE Trans. Inform. Theory 37 (6) (1991) 1567-1576.

[21] R. Stanley, Hilbert functions of graded algebras, Adv. Math. 28 (1978) $57-83$.

[22] H. Stichtenoth, Algebraic function fields and codes (Universitext, Springer-Verlag, Berlin, 1993).

[23] S. Tohăneanu, Lower bounds on minimal distance of evaluation codes, Appl. Algebra Engrg. Comm. Comput. 20 (5-6) (2009) 351-360.

[24] M. Tsfasman, S. Vladut and D. Nogin, Algebraic geometric codes: basic notions (Mathematical Surveys and Monographs 139, American Mathematical Society, Providence, RI, 2007).

[25] R. H. Villarreal, Monomial Algebras (Monographs and Textbooks in Pure and Applied Mathematics 238, Marcel Dekker, New York, 2001).

Manuel GONZÁLEZ SARABIA,

Departamento de Ciencias Básicas,

Unidad Profesional Interdisciplinaria en

Ingeniería y Tecnologías Avanzadas,

Instituto Politécnico Nacional,

07340, México, D.F.

Partially supported by COFAA-IPN and SNI.

Email: mgonzalezsa@ipn.mx 
Carlos RENTERÍA MÁRQUEZ,

Departamento de Matemáticas,

Escuela Superior de Física y Matemáticas,

Instituto Politécnico Nacional,

07300, México, D.F.

Partially supported by COFAA-IPN and SNI.

Email: renteri@esfm.ipn.mx

Eliseo SARMIENTO ROSALES,

Departamento de Matemáticas,

Escuela Superior de Física y Matemáticas,

Instituto Politécnico Nacional,

07300, México, D.F.

Partially supported by SNI.

Email: esarmiento@ipn.mx 